\title{
Persistent Neutropenia after ABOi Kidney Transplantation: A Case Report
}

\author{
Gabriele Gualtiero Andenna ${ }^{1}$, Marilena Gregorini ${ }^{1,2, *}$, Chiara Elena ${ }^{3}$, Miriam Fusi ${ }^{1}$, Rosa Colangelo ${ }^{1}$, \\ Eleonora Francesca Pattonieri ${ }^{1}\left(\mathbb{0}\right.$, Maria Antonietta Grignano ${ }^{1}{ }^{(\mathbb{D}}$, Carmelo Libetta $^{1,2}$ and Teresa Rampino ${ }^{1}$ \\ 1 Nephrology, Dialysis and Transplant Unit, San Matteo Foundation IRCCS, University of Pavia, \\ Piazzale Golgi 2, 27100 Pavia, Italy; andenna.gabriele@gmail.com (G.G.A.); miriam.fusi@live.it (M.F.); \\ rossana_colangelo@hotmail.it (R.C.); ef.pattonieri@gmail.com (E.F.P.); wta87@hotmail.it (M.A.G.); \\ carmelo.libetta@unipv.it (C.L.); t.rampino@smatteo.pv.it (T.R.) \\ 2 Department of Internal Medicine and Therapeutics, San Matteo Foundation IRCCS, University of Pavia, \\ 27100 Pavia, Italy \\ 3 Haematology and Bone Marrow Transplant Unit, San Matteo Foundation IRCCS, University of Pavia, \\ 27100 Pavia, Italy; c.elena@smatteo.pv.it \\ * Correspondence: mgregorini@hotmail.com
}

check for updates

Citation: Andenna, G.G.; Gregorini, M.; Elena, C.; Fusi, M.; Colangelo, R.; Pattonieri, E.F.; Grignano, M.A.; Libetta, C.; Rampino, T. Persistent Neutropenia after ABOi Kidney Transplantation: A Case Report. Transplantology 2021, 2, 183-190. https://doi.org/10.3390/ transplantology2020017

Academic Editor: Lionel Rostaing

Received: 17 March 2021

Accepted: 13 May 2021

Published: 16 May 2021

Publisher's Note: MDPI stays neutral with regard to jurisdictional claims in published maps and institutional affiliations.

Copyright: (c) 2021 by the authors. Licensee MDPI, Basel, Switzerland. This article is an open access article distributed under the terms and conditions of the Creative Commons Attribution (CC BY) license (https:// creativecommons.org/licenses/by/ $4.0 /)$.

\begin{abstract}
Post-transplant neutropenia (PTN) is frequently reported in the first-year after transplantation. Although prevalence and clinical consequences are widely described, there are no guidelines to manage diagnosis and treatment. We report here a case of persistent PTN occurred in a patient undergoing a kidney transplant from an AB0-incompatible living donor. The desensitization protocol consisted of Rituximab administration and immunoadsorption while the pre-transplant protocol, which was initiated 14 days before the transplant, included Tacrolimus, Mofetil Mycophenolate (MMF), antimicrobial and antiviral prophylaxis. Induction therapy consisted of anti-thymocyte globulins and steroids, while maintenance after transplantation consisted of steroid, tacrolimus and MMF. When the first occurrence of leukopenia was observed six weeks after the transplant, firstly antimicrobial/antiviral prophylaxis was stopped and later also MMF treatment was interrupted but severe neutropenia relapsed after MMF resuming treatment. Immunological and virological causes were excluded. The patient was treated with Filgrastim. Bone marrow biopsy, which was performed to exclude a hematological cause of severe persistent neutropenia, revealed a bone marrow hypoplasia with neutrophils maturation interrupted at the early stages. This case highlights the need to establish diagnostic and therapeutic guidelines for PTN which take in consideration all the therapeutic steps including the pre-transplant phase in particular in the context of ABOi where immunosuppression is more consistent.
\end{abstract}

Keywords: neutropenia; AB0i; kidney transplantation; bone marrow biopsy; ATG; CMV

\section{Introduction}

Neutropenia, defined as an absolute neutrophil count (ANC) lower than 1500 cells $/ \mu \mathrm{L}$ $\left(<1.5 \times 10^{9} / \mathrm{L}\right)$, is a common side effect of solid organ transplantation in adults [1-5] and in the pediatric population [6].

Post-transplant neutropenia (PTN) occurs mainly during the first year after transplantation, with an incidence ranging from $10 \%$ to $44 \%$ as estimated by retrospective studies $[1-5,7]$.

PTN potentially increases the risk of infections in vulnerable patients but also exposes them to higher risks of rejection due to the extended suspension of mycophenolate mofetil (MMF) treatment [1].

PTN is predominantly caused by drug toxicity induced by immunosuppressive treatment (i.e., lymphocyte depleting agents, antimetabolic agents, tacrolimus), or antiinfectious agents, such as valganciclovir (VGCV) and trimethoprim-sulfamethoxazole 
(TMP/SMX) [8,9], while viral infections such as cytomegalovirus (CMV), Epstein-Barr virus (EBV), herpes simplex virus 1,2 or parvovirus B 19 are the main causes of infectious neutropenia $[10,11]$.

Currently, there are no guidelines for managing PTN in solid organ transplant recipients.

Here, we report a case of neutropenia in a renal transplant recipient from a living $\mathrm{AB} 0$ incompatible (AB0i) donor. A multidisciplinary approach allowed us to identify PTN's underlying cause and guided therapeutic management.

\section{Case Report}

A blood group zero male patient (56 years old) with end stage renal disease due to IgA nephropathy underwent a kidney transplant from an ABO-incompatible (AB0i) living donor. For the desensitization protocol the patient received one dose of Rituximab (RTX-Mundipharma Pharmaceuticals S.R.L. Milano, Italy) $(375 \mathrm{mg} / \mathrm{sqm}) 2$ weeks before the scheduled date of transplantation. However, the planned transplant was postponed for 3 months due to the onset of COVID-19 pandemic in Italy. Since blood peripheral CD19+ cells were undetectable prior to transplant, a second RTX infusion was not performed.

To remove anti-A antibodies, whose titer before transplant was 1:8, the patient underwent 3 sessions of immunoadsorption with Globaffin column (Fresenius Medical CareSankt Wendel, DE) until the anti-A antibody titer was below 1:2. The pre-transplant immunosuppressive protocol, which included Tacrolimus (4 mg every twelve hours) and Mofetil Mycophenolate (MMF $1 \mathrm{~g}$ every twelve hours), started 14 days prior to the transplant. Concomitantly, antiviral prophylaxis with Acyclovir (400 mg every twelve hours adjusted to creatinine clearance value) was started.

Induction therapy consisted of Antithymocyte Globulins (ATG-Fresenius Biotech $\mathrm{GmbH}$, Munich, Germany) (total dose $5 \mathrm{mg} / \mathrm{kg}$ ) and steroids, while immunosuppression after transplantation consisted of steroid, tacrolimus (through levels $12-14 \mathrm{ng} / \mathrm{mL}$ ) and MMF (1 g every twelve hours).

The recipient was not pre-immunized and shared 3 matches with his donor (1 being HLA-A and 2 being in HLA DQB); furthermore, there was a mismatch D/R for CMV and Toxoplasma Gondii, wherein the recipient was negative for both.

Antiviral prophylaxis with acyclovir (400 mg every twelve hours) and valganciclovir (450 mg every twelve hours adjusted to creatinine clearance value) was scheduled for the first six months after transplantation.

The onset of post-transplant diabetes mellitus requiring insulin treatment occurred during the first month after transplantation.

The first occurrence of leukopenia was observed six weeks after the transplant and TMP/SPX was interrupted immediately and soon after antiviral prophylaxis and MMF were interrupted.

Frequent follow up visits were planned to monitor the clinical, bio-humoral, immunological and infective course of the observed condition.

Ten weeks after transplantation, complete blood count was normalized and MMF (750 mg BID) and TMP/SMX were resumed. However, after 15 days, severe neutropenia [10] (absolute neutrophils count $<500$ cells $/ \mathrm{mm}^{3}$ ) occurred and these drugs were discontinued.

Due to persistence of severe neutropenia, the patient had to be treated with subcutaneous (sc) Filgrastim ( 2 sc doses of $30 \mathrm{MU}$, the second 3 days apart), resulting in a rise in the neutrophil count.

Immunological and virological monitoring, performed every two weeks, did not show the appearance of anti-A antibodies, nor viraemia for CMV, EBV, parvovirus B19, polyoma BK, HIV, herpes viridae 6 and 8 ever appeared.

Avidity test for Toxoplasma and detection of anti-donor specific antibodies were carried out based on center protocol schedule, always providing negative results.

Anemia, thrombocytopenia, infections and fever never occurred during this time frame and kidney function was normal. As neutrophils raised, MMF was resumed albeit 
at a reduced dose (500 mg every twelve hours) and Pneumocystis Jiroveci Pneumonia (PCP)/toxoplasma (TOXO) (PCP-TOXO) prophylaxis was performed with Atovaquone, due to its reduced effect on neutrophil count.

However, a few days later, neutropenia occurred again. Hence, immunosuppressive therapy was limited to tacrolimus and Methylprednisolone (MP) with supplementation of folic acid and group's B vitamins. However, neutrophil counts did not improve and reached a minimum of 130 neutrophils/ $\mu \mathrm{L} 5$ months after transplant.

This relapse required a second administration of Filgrastim (2 sc doses of $30 \mathrm{MU}$ 3 days apart). Despite an initially positive response to granulokine therapy, leukocytes and neutrophils count decreased again. Neither CMV nor EBV viremia were detected. A complete hematological work-up was conducted, that allowed to exclude any cause of secondary neutropenia: B12, folate, iron, copper deficiency, viral tests, autoimmune screening, thyroid function tests, anti-granulocytes antibodies. Flow cytometry on peripheral blood sample excluded a clonal large granular lymphocyte (LGL) population. Therefore, the patient underwent a bone marrow biopsy in order to exclude a hematological cause of neutropenia.

The BM trephine biopsy (BMB) showed a hypocellular bone marrow (cellularity 30\%), without significant dysplasia and no increased amount of blasts. Karyotype was normal $(46, \mathrm{XY})$ and the morphological evaluation of bone marrow aspirate did not show relevant dyserithropoiesis, dysmegakariopoiesis or dysgranulopoiesis. Neutrophils maturations series was severely underrepresented and myeloid cellularity appeared in the early stages of maturation.

Genomic analysis with next-generation sequencing (NGS) (Illumina Myeloid Panel) did not show any oncogenic somatic mutation.

Since no viremia for CMV, EBV, HIV, Parvovirus B19, Herpes virus 6 and 8 was detected in peripheral blood, no further analysis was performed on the bone marrow.

The timeline of events is summarized in Figure 1 and the diagnostic algorithm is described in Figure 2. 


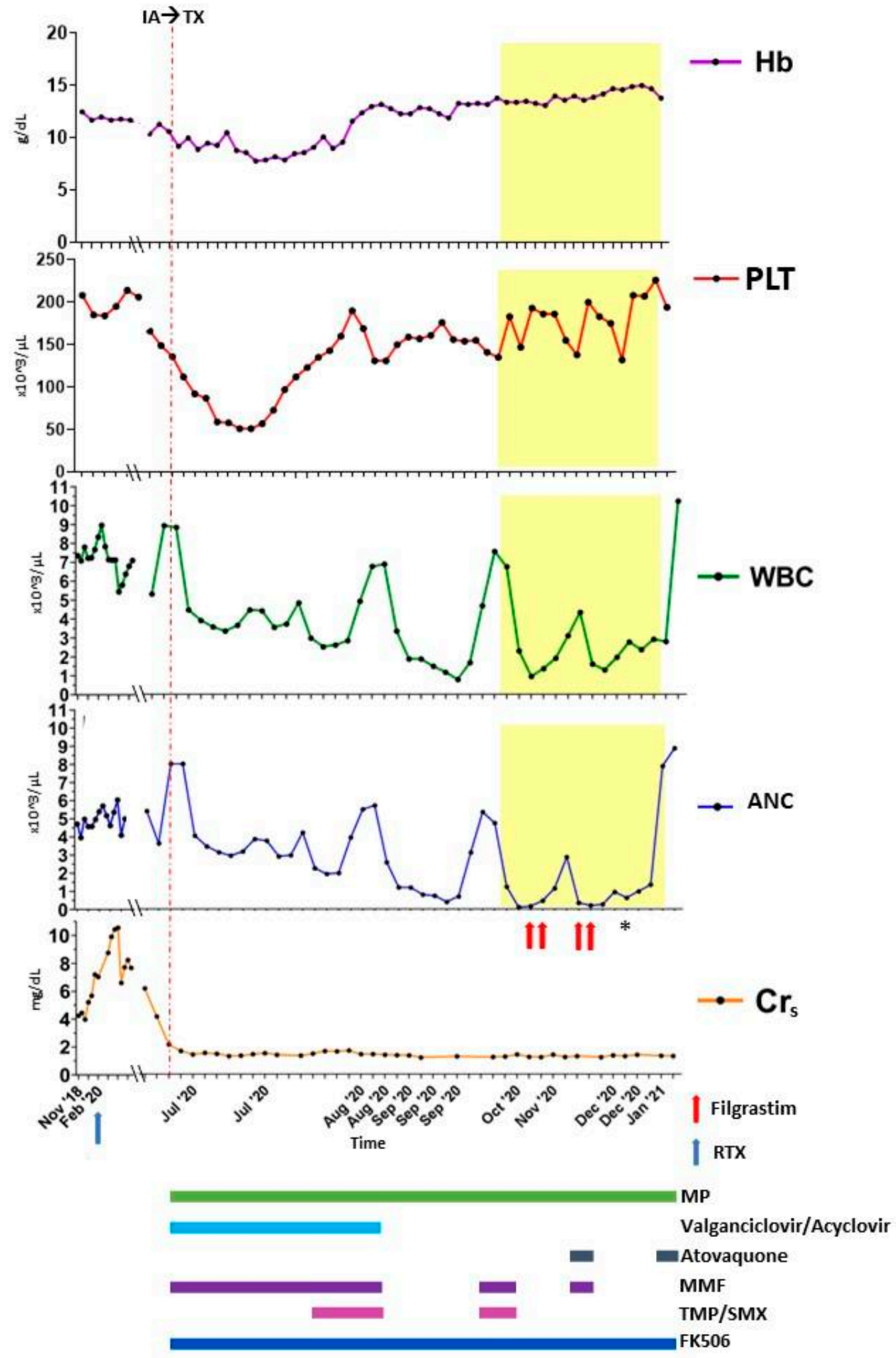

* BMB

Figure 1. Clinical and biochemical timeline of case report, WBC: white blood cells, PLT: platelets, ANC: absolute neutrophil count, Crs: serum creatinine, MP: methylprednisolone, MMF: mycophenolate mofetil, TMP/SMX: trimethoprim/sulfamethoxazole, FK506: tacrolimus, RTX: rituximab, BMB: bone marrow biopsy, TX: transplantation, IA: immunoadsorption. 


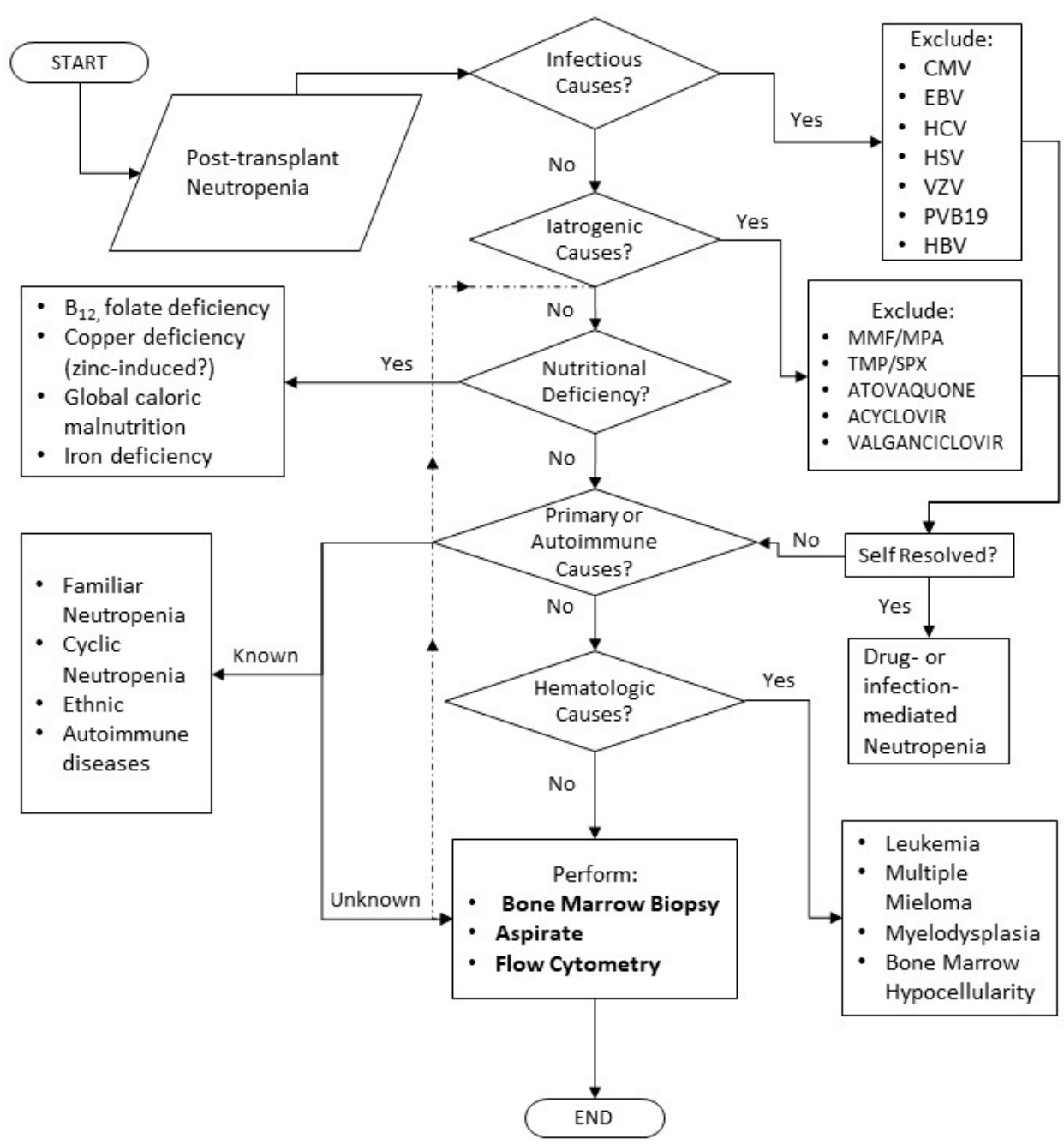

Figure 2. The diagnostic algorithm conceived and applied to the clinical case described.

\section{Discussion}

Neutropenia is a frequent occurrence in the first year following kidney transplantation with an incidence range of $15-48 \%$ [1]. However, since, at present, there are no guidelines for diagnosis and treatment of PTN, an empirical approach to treat this condition has been suggested.

The present case report aims to describe the diagnostic steps and the therapeutic approach followed in a patient with moderate-severe neutropenia occurring in the first 6 months post-transplant.

It is acknowledged that neutropenia is predominantly of iatrogenic origin in kidney allograft recipients, since the medications used in the induction and maintenance of immunosuppressive therapy or Angiotensig-converting-enzyme inhibitors and proton pump inhibitors determine myelosuppression $[10,12,13]$. In order to exclude an iatrogenic cause in the case herein reported, antiviral drugs and PCP-TOXO chemoprophylaxis were suspended in the first instance, resulting in an immediate rise in neutrophil count. However, the neutropenia persisted and required the suspension of MMF. Meanwhile, viral infections causing leukopenia (CMV-EBV-HerpesViridae, Parvovirus B19) were excluded.

Administration of myelosensitive growth factors determined a transient remission of neutropenia, but the persistent leukopenia prompted us to investigate whether this condition was caused by any rare anti-neutrophil antibodies-mediated autoimmune disease, or was an infrequent form of tacrolimus neutropenia [14-17]. 
We considered both causes unlikely, since autoimmune disease occurrence during immunosuppressive therapy and tacrolimus neutropenia are remote events.

No anti-neutrophil antibodies were detected. In parallel, BMB and needle aspiration were performed to exclude a bone marrow-dependent cause of neutropenia and to assess the patient's hematopoietic response to post-transplant immunosuppressive therapy, which is known to be contingent on the individual bone marrow reserve. At present, no noninvasive tests are available to explore the bone marrow reserve, apart from needle aspiration and $\mathrm{BMB}$ and it is remarkable that no investigation on bone marrow reserve is performed in patients waiting for kidney transplant.

However, bone marrow hypoplasia and myelodysplastic syndrome occur more frequently in patients affected by chronic renal failure as compared to the general population and this condition also increases with age and with dialysis duration [18,19].

Therefore, it is reasonable that a reduced bone marrow reserve could be common in patients in the kidney transplant waiting list and that these subjects could develop moderate-severe leukoneutropenia when treated with a standard immunosuppressive therapy combined with drugs with myelosuppressive effects such as antivirals and antimicrobials.

We consider that the above scenario best describes the findings observed in our case, wherein leukocyte count lay within normal limits before the transplant and was found to be severely compromised following drugs administered immediately after the transplant.

Hence, we conclude that drug treatment can compromise a borderline bone marrow reserve, leading to severe leukoneutropenia, with the ultimate effect of increasing the risk of infections, considering that neutropenia and lymphocytopenia often coexist [20].

$\mathrm{BMB}$ and needle aspiration in the case herein described excluded neoplastic forms but showed an inadequate bone marrow reserve, since the neutrophils maturation series was severely underrepresented and myeloid cellularity appeared in the early stages of maturation.

$\mathrm{BMB}$ is not required in transient and mild post-transplant neutropenia, but it can become an essential diagnostic tool for detecting persistent or severe forms of neutropenia.

There are currently no guidelines for diagnosing and managing post-transplant neutropenia even if therapeutic management of graft recipients with neutropenia is challenging, since the therapeutic regimen needs to balance the infectious risk and rejection risk $[20,21]$.

In this case Filgastrim was employed, because neutropenia was severe, associated with lymphocytopenia and it did not regress upon discontinuation of the antimetabolites. This therapeutic scheme allowed us to manage it in outpatient and to prevent infectious complications [22]. Remarkably, despite donor/recipient mismatch with regard to CMV and Toxoplasma, the patient presented no symptoms of disease.

Although there are no clear guidelines, we believe that educating the patient on health and hygiene practices to be held at home is pivotal in the therapeutic approach. Such an approach should avoid causing excessive concern and alarm in the patient.

Furthermore, training the patient to recognize and report the first signs of infection is of the utmost importance. Likewise, the relevance of oral and hand hygiene needs to be stressed, while sterilizing the environment is dispensable.

It is acknowledged that prophylactic use of antibiotics did not prove useful in this context, while administration of myeloid growth factors (G-CSF; GM-CSF) was effective in preventing infectious complications in numerous cases [23-25].

The diagnostic workup performed in this patient did not include MMF, copper and zinc serum level measurement and ACE serum level being sarcoidosis a rare cause of neutropenia.

Since its introduction into clinical practice, mycophenolate (MPA) has been considered as a drug not requiring individual therapeutic monitoring. Therefore, the role of therapeutic drug monitoring (TDM) for MPA is still controversial. 
It is acknowledged that the average free fraction of MPA is increased in kidney transplant recipients with lower eGFR and it is also reported that a high MPA free fraction is associated with leukopenia [26].

However, due to the complexity associated to MPA pharmacokinetics, the challenges in fully establishing an MPA concentration-toxicity relationship and the conflicting evidence supporting TDM-guided dosing over standardized dosing, TDM is not currently considered as a routine practice [27].

\section{Conclusions}

This case emphasizes the need to detect via pre-transplantation screening patients at risk of PTN in order to customize how immunosuppression can be properly induced.

Our proposal is to thoroughly evaluate during the pre-transplant screening the blood count and investigate whether the patient experienced previous episodes of leukopenia or pancytopenia occurred.

We also recommend to identify potential vitamins or minerals deficiencies; if the leuco/cytopenia persists after vitamin supplementation then we propose to perform a bone marrow biopsy to investigate further.

An interesting future development in neutropenia assessment is the screening of patients for somatic mutations which may be associated to late neutropenia onset. Such research is under exploration.

We also highlight the need to establish diagnostic and therapeutic guidelines for PTN in post transplantation, as well as to provide guidance in the context of iatrogenic neutropenia after chemotherapy.

This becomes even more relevant in incompatible $\mathrm{AB} 0$ transplants, where immunosuppression is more consistent and where neutropenia can affect $50 \%$ of cases and can have a prolonged duration. Looking back at our diagnostic path we believe that BMB should be considered among the first investigations to be carried out and before administering growth factors whose contraindication would be the presence of myeloid leukemia.

Author Contributions: G.G.A. writing original draft preparation; M.G. writing review and editing; C.E. revision and editing; M.F. methodology; R.C. visualization; E.F.P. writing review and editing; M.A.G. visualization; C.L. supervision; T.R. review, editing and supervision. All authors have read and agreed to the published version of the manuscript.

Funding: This research received no external funding.

Institutional Review Board Statement: Not applicable.

Informed Consent Statement: Written informed consent has been obtained from the patient to publish this paper.

Data Availability Statement: Not applicable.

Conflicts of Interest: The authors declare no conflict of interest.

\section{References}

1. Zafrani, L.; Truffaut, L.; Kreis, H.; Etienne, D.; Rafat, C.; Lechaton, S.; Anglicheau, D.; Zuber, J.; Ciroldi, M.; Thervet, E.; et al. Incidence, risk factors and clinical consequences of neutropenia following kidney transplantation: A retrospective study. Am. J. Transpl. 2009, 9, 1816-1825. [CrossRef] [PubMed]

2. Alraddadi, B.; Nierenberg, N.E.; Price, L.L.; Chow, J.K.; Poutsiaka, D.D.; Rohrer, R.J.; Cooper, J.T.; Freeman, R.B.; Snydman, D.R. Characteristics and outcomes of neutropenia after orthotopic liver transplantation. Liver Transpl. 2016, 22, 217-225. [CrossRef] [PubMed]

3. Rosengarten, D.; Fakterman, M.; Raviv, Y.; Rusanov, V.; Fox, B.; Kramer, M. Neutropenia Following Lung Transplantation. J. Hear Lung Transplant. 2015, 34, S255. [CrossRef]

4. Chow, J.K.; Ruthazer, R.; Boucher, H.W.; Nierenberg, N.; Vest, A.R.; DeNofrio, D.; Snydman, D.R. Factors Associated with Neutropenia Post-Heart Transplantation. J. Hear Lung Transpl. 2020, 39, S482. Available online: https:/ / www.sciencedirect.com/ science/article/pii/S1053249820300577 (accessed on 15 May 2021). [CrossRef]

5. Hartmann, E.L.; Gatesman, M.; Roskopf-Somerville, J.; Stratta, R.; Farney, A.; Sundberg, A. Management of leukopenia in kidney and pancreas transplant recipients. Clin. Transplant. 2008, 22, 822-828. [CrossRef] [PubMed] 
6. $\quad$ Rose-Felker, K.; Mukhtar, A.; Kelleman, M.S.; Deshpande, S.R.; Mahle, W.T. Neutropenia in pediatric heart transplant recipients. Pediatr. Transpl. 2018, 22, e13130. [CrossRef]

7. Barry, M.; Chandra, S.; Hymes, K.B. Principles and Practice of Transplant Infectious Diseases. Princ. Pract. Transpl. Infect. Dis. 2019, 10, 199-207.

8. Brum, S.; Nolasco, F.; Sousa, J.; Ferreira, A.; Possante, M.; Pinto, J.R.; Barroso, E.; Santos, J.R. Leukopenia in Kidney Transplant Patients with the Association of Valganciclovir and Mycophenolate Mofetil. Transpl. Proc. 2008, 40, 752-754. Available online: https://www.sciencedirect.com/science/article/pii/S0041134508001577 (accessed on 15 May 2021). [CrossRef]

9. Keisu, M.; Wiholm, B.-E.; Palmblad, J. Trimethoprim-sulphamethoxazole-associated blood dyscrasias. Ten years' experience of the Swedish spontaneous reporting system. J. Intern. Med. 1990, 228, 353-360. [CrossRef]

10. Newburger, P.E.; Dale, D.C. Evaluation and Management of Patients with Isolated Neutropenia. Semin Hematol. 2013, 50, 198-206. [CrossRef]

11. Martín-Gandul, C.; Pérez-Romero, P.; González-Roncero, F.M.; Berdaguer, S.; Gómez, M.A.; Lage, E.; Sánchez, M.; Cisneros, J.M.; Cordero, E. Clinical impact of neutropenia related with the preemptive therapy of CMV infection in solid organ transplant recipients. J. Infect. 2014, 69, 500-506. [CrossRef]

12. Gladziwa, U.; Hermans, M.M.H.; Naami, A.; Maastricht, A.H. Unexpected rate of severe leucopenia with the association of mycophenolate mofetil and valganciclovir in kidney transplant recipients. Nephrol. Dial. Transplant. 2007, 22, 671-672.

13. Hong, J.C.; Kahan, B.D. Sirolimus-Induced Thrombocytopenia and Leukopenia in Renal Transplant Recipients: Risk Factors, Incidence, Progression, and Management. Transplantation 2000, 69, 2085-2090. [CrossRef] [PubMed]

14. De Rycke, A.; Dierickx, D.; Kuypers, D.R. Tacrolimus-induced neutropenia in renal transplant recipients. Clin. J. Am. Soc. Nephrol. 2011, 6, 690-694. [CrossRef] [PubMed]

15. Clément, J.; Taton, B.; Merville, P.; Viallard, J.F.; Lazaro, E.; Couzi, L.; Rivière, E. Two cases of tacrolimus-induced neutropenia: A probably diagnosed cause of neutropenia after solid-organ transplantation. Clin. Transplant. 2018, 32, e13295. [CrossRef] [PubMed]

16. Boxer, L.A.; Bolyard, A.A.; Marrero, T.M.; Tran, E.L.; Bonilla, M.A.; Newburger, P.E.; Dale, D.C. Is There a Role for Anti-Neutrophil Antibody Testing in Predicting Spontaneous Resolution of Neutropenia in Young Children. Blood 2015, 126, 2211. [CrossRef]

17. Akhtari, M.; Curtis, B.; Waller, E.K. Autoimmunity Reviews Autoimmune neutropenia in adults. Autoimmun. Rev. 2009, 9, 62-66. [CrossRef]

18. Chang, M.Y.; Lin, S.F.; Wu, S.C.; Yang, W.C. Myelodysplastic syndrome: The other cause of anemia in end-stage renal disease patients undergoing dialysis. Sci. Rep. 2020, 10, 1-9.

19. Weng, C.H.; Lu, K.Y.; Hu, C.C.; Huang, W.H.; Wang, I.K.; Yen, T.H. Bone Marrow Pathology Predicts Mortality in Chronic Hemodialysis Patients. BioMed Res. Int. 2015, 2015, 160382. [CrossRef]

20. Brown, A.E. Neutropenia, Fever, and Infection. Am. J. Med. 1984, 76, 421-428. [CrossRef]

21. Knoll, G.A.; MacDonald, I.; Khan, A.; Van Walraven, C. Mycophenolate mofetil dose reduction and the risk of acute rejection after renal transplantation. J. Am. Soc. Nephrol. 2003, 14, 2381-2386. [CrossRef]

22. Schmaldienst, S.; Bekesi, G.; Deicher, R.; Franz, M.; Hörl, W.H.; Pohanka, E. Recombinant human granulocyte colony-stimulating factor after kidney transplantation: A retrospective analysis to evaluate the benefit or risk of immunostimulation. Transplantation 2000, 69, 527-531. [CrossRef]

23. Davidson, M.; Min, Y.I.; Holbrook, J.T.; Van Natta, M.L.; Murphy, R.; Jabs, D.A.; Welch, W.; Meinert, C.L. Use of filgrastim as adjuvant therapy in patients with AIDS-related cytomegalovirus retinitis. AIDS 2002, 16, 757-765. [CrossRef] [PubMed]

24. Kuritzkes, D.R.; Parenti, D.; Ward, D.J.; Rachlis, A.; Wong, R.J.; Mallon, K.P.; Rich, W.J.; Jacobson, M.A. Filgrastim prevents severe neutropenia and reduces infective morbidity in patients with advanced HIV infection: Results of a randomized, multicenter, controlled trial. Aids 1998, 12, 65-74. [CrossRef]

25. Gregorini, M.; Castello, M.; Rampino, T.; Bosio, F.; Bedino, G.; Esposito, P.; Borroni, G.; Dal Canton, A. GM-CSF contributes to prompt healing of ecthyma gangrenosum lesions in kidney transplant recipient. J. Nephrol. 2012, 25, 137-139. [CrossRef] [PubMed]

26. Meier-Kriesche, H.U.; Shaw, L.M.; Korecka, M.; Kaplan, B. Pharmacokinetics of mycophenolic acid in renal insufficiency. Ther. Drug Monit. 2000, 22, 27-30. [CrossRef] [PubMed]

27. Kaplan, B.; Meier-Kriesche, H.U.; Friedman, G.; Mulgaonkar, S.; Gruber, S.; Korecka, M.; Brayman, K.L.; Shaw, L.M. The effect of renal insufficiency on mycophenolic acid protein binding. J. Clin. Pharmacol. 1999, 39, 715-720. [CrossRef] 